DOI: $10.17516 / 1997-1397-2020-13-3-360-372$

УДК $517.765+512.816$

\title{
On the Orbits of Nilpotent 7-dimensional Lie Algebras in 4-dimensional Complex Space
}

\author{
Alexander V. Loboda* \\ Voronezh State Technical University \\ Voronezh, Russian Federation \\ Ripsime S. Akopyan ${ }^{\dagger}$ \\ MIREA, Russian Technological University \\ Moscow, Russian Federation \\ Vladislav V. Krutskikh ${ }^{\ddagger}$ \\ Voronezh State University \\ Voronezh, Russian Federation
}

Received 10.02.2020, received in revised form 10.03.2020, accepted 20.04.2020

\begin{abstract}
We study one-parameter families of 7-dimensional nilpotent indecomposable Lie algebras and the orbits of holomorphic realizations of such algebras in a 4-dimensional complex space. It is shown, in contrast to the orbits of 5-dimensional nilpotent Lie algebras in 3-dimensional case, that two such families (out of the existing nine ones) admit orbits that are Levi non-degenerate (homogeneous) real non-spherical hypersurfaces. Up to holomorphic equivalence, all obtained non-degenerate nonspherical orbits are graphs of polynomials of degree 4.
\end{abstract}

Keywords: Lie algebra, complex space, vector field, holomorphic function, homogeneous variety, Levi degeneracy.

Citation: A.V.Loboda, R.S.Akopyan, V.V.Krutskikh, On the Orbits of Nilpotent 7-dimensional Lie Algebras in 4-dimensional Complex Space, J. Sib. Fed. Univ. Math. Phys., 2020, 13(3), 360-372.

DOI: 10.17516/1997-1397-2020-13-3-360-372.

\section{Introduction}

In connection with the problem of describing holomorphically homogeneous real hypersurfaces of 3-dimensional complex space the technique was developed in the articles [1-4] for studying holomorphic realizations of 5 -dimensional Lie algebras in the space $\mathbb{C}^{3}$.

Under this, according to the result of [2], the orbits of nilpotent 5-dimensional Lie algebras in the space $\mathbb{C}^{3}$ can be either Levi degenerate hypersurfaces, or non-degenerate spherical ones, i.e. holomorphic images of non-degenerate quadrics

$$
\operatorname{Im} z_{3}=\left|z_{1}\right|^{2} \pm\left|z_{2}\right|^{2} .
$$

In the present work, a similar technique is used for study of holomorphically homogeneous hypersurfaces in the space $\mathbb{C}^{4}$. In particular, below we consider the orbits of holomorphic real-

*lobvgasu@yandex.ru https://orcid.org/0000-0002-0285-5841

†akrim111@yandex.ru

${ }^{\ddagger}$ krutskihvlad@mail.ru

(c) Siberian Federal University. All rights reserved 
izations in this space of nilpotent 7-dimensional Lie algebras (representations of these algebras in the algebra of germs of holomorphic vector fields in $\mathbb{C}^{4}$ ).

Note that situations with 5-dimensional and 7-dimensional Lie algebras significantly differ from each other in the number of algebraically different objects. So, according to [5], there are only 9 nilpotent 5 -dimensional Lie algebras (6 indecomposable and 3 decomposable). And the family of 7-dimensional indecomposable Lie algebras contains (see [6]) 140 isolated nilpotent algebras and 9 one-parameter families of nilpotent Lie algebras. In [6], these families are denoted by

$$
147 E, 1357 M, 1357 N, 1357 S, 12457 N, 123457 I, 147 E_{1}, 1357 Q R S_{1}, 12457 N_{2} .
$$

Below 6 such families are considered with the aim of comparing situations with holomorphic homogeneity of hypersurfaces in 3-dimensional and 4-dimensional complex spaces. The main interest of the article is related to nondegenerate orbits of Lie algebras; the main results are presented in the following two theorems.

Theorem 1. Each 7-dimensional orbit in the space $\mathbb{C}^{4}$ of any algebra of four families $1357 N$, $12457 N$, 123457I, 12457 $N_{2}$ must be degenerate in Levi sense.

Theorem 2. The two families $1357 M, 1357 Q R S_{1}$ have algebras, admitting holomorphic realizations with Levi non-degenerate non-spherical 7-dimensional orbits in the space $\mathbb{C}^{4}$. Up to holomorphic equivalence, all such orbits of the $1357 M$ family are described by the formulas

$$
y_{4}=y_{1} y_{3}+y_{2}^{2}+y_{1}^{2} y_{2}+D y_{1}^{4}, \quad D \neq \frac{1}{12}
$$

among Levi non-degenerate nonspherical orbits of the $1357 Q R S_{1}$ family there are holomorphic images of the surfaces

$$
y_{4}=y_{1} y_{3}+y_{2}^{2}+x_{1} y_{1} y_{2}+D y_{1}^{4}, \quad D \neq \frac{1}{12} .
$$

The families $1357 S, 147 E, 147 E_{1}$ also contain algebras whose orbits in $\mathbb{C}^{4}$ are non-degenerate nonspherical hypersurfaces. However, all such surfaces are described by formula (2). Due to the limited scope of the article, we do not discuss these three families.

\section{Families of 7-dimensional nilpotent Lie algebras}

The existence of the families of nilpotent algebras depending on the real parameter, distinguishes the cases of 7-dimensional algebras and 5-dimensional ones. We denote this parameter by the common symbol $\lambda$ and discuss the families of 7-dimensional indecomposable algebras mentioned above. Each of them is described in some basis $e_{1}, \ldots, e_{7}$ by the following relationships:

$$
\begin{gathered}
1357 M(\lambda \neq 0):\left[e_{1}, e_{2}\right]=e_{3},\left[e_{1}, e_{3}\right]=e_{5},\left[e_{1}, e_{4}\right]=e_{6},\left[e_{1}, e_{5}\right]=e_{7}, \\
{\left[e_{2}, e_{4}\right]=e_{5},\left[e_{2}, e_{6}\right]=\lambda e_{7},\left[e_{3}, e_{4}\right]=(1-\lambda) e_{7} .} \\
1357 N:\left[e_{1}, e_{2}\right]=e_{3},\left[e_{1}, e_{3}\right]=e_{5},\left[e_{1}, e_{4}\right]=e_{6},\left[e_{1}, e_{5}\right]=e_{7}, \\
{\left[e_{2}, e_{3}\right]=\lambda e_{7},\left[e_{2}, e_{4}\right]=e_{5},\left[e_{3}, e_{4}\right]=e_{7},\left[e_{4}, e_{6}\right]=e_{7} .} \\
12457 N:\left[e_{1}, e_{2}\right]=e_{3},\left[e_{1}, e_{3}\right]=e_{4},\left[e_{1}, e_{4}\right]=e_{7},\left[e_{1}, e_{5}\right]=e_{6},\left[e_{1}, e_{6}\right]=e_{7}, \\
{\left[e_{2}, e_{3}\right]=e_{5},\left[e_{2}, e_{4}\right]=e_{6},\left[e_{2}, e_{5}\right]=\lambda e_{7},\left[e_{2}, e_{6}\right]=e_{7},\left[e_{3}, e_{4}\right]=e_{7},\left[e_{3}, e_{5}\right]=-e_{7} .} \\
123457 I:\left[e_{1}, e_{2}\right]=e_{3},\left[e_{1}, e_{3}\right]=e_{4},\left[e_{1}, e_{4}\right]=e_{5},\left[e_{1}, e_{5}\right]=e_{6},\left[e_{1}, e_{6}\right]=e_{7}, \\
{\left[e_{2}, e_{3}\right]=e_{5},\left[e_{2}, e_{4}\right]=e_{6},\left[e_{2}, e_{5}\right]=\lambda e_{7},\left[e_{3}, e_{4}\right]=(1-\lambda) e_{7} .}
\end{gathered}
$$




$$
\begin{array}{r}
1357 Q R S_{1}(\lambda \neq 0):\left[e_{1}, e_{2}\right]=e_{3},\left[e_{1}, e_{3}\right]=e_{5},\left[e_{1}, e_{4}\right]=e_{6},\left[e_{1}, e_{5}\right]=e_{7}, \\
{\left[e_{2}, e_{3}\right]=-e_{6},\left[e_{2}, e_{4}\right]=e_{5},\left[e_{2}, e_{6}\right]=\lambda e_{7},\left[e_{3}, e_{4}\right]=(1-\lambda) e_{7} .} \\
12457 N_{2}:\left[e_{1}, e_{2}\right]=e_{3},\left[e_{1}, e_{3}\right]=e_{4},\left[e_{1}, e_{4}\right]=-e_{6},\left[e_{1}, e_{5}\right]=e_{7},\left[e_{1}, e_{6}\right]=e_{7}, \\
{\left[e_{2}, e_{3}\right]=e_{5},\left[e_{2}, e_{4}\right]=e_{7},\left[e_{2}, e_{5}\right]=-e_{6}+\lambda e_{7},\left[e_{3}, e_{5}\right]=-e_{7} .}
\end{array}
$$

The main point of [1-4], as well as of this article, is the use of large Abelian subalgebras of studied algebras and simplification of bases of these subalgebras.

It can be noted that for nilpotent Lie algebras of arbitrary dimensions the existence of such subalgebras (and even Abelian ideals) follows from Morozov's known statement [7].

In the case of algebras from the families (4)-(9) it is easy to make sure that each of them contains a 4-dimensional Abelian ideal with the following basis:

$$
\begin{aligned}
& 12457 N, 12457 N_{2}, 123457 I: I_{4}=<e_{4}, e_{5}, e_{6}, e_{7}>, \\
& 1357 M, 1357 N, 1357 Q R S_{1}: I_{4}^{\prime}=<e_{3}, e_{5}, e_{6}, e_{7}>.
\end{aligned}
$$

Remark. For some of the families (4)-(9), each algebra contains several different 4-dimensional Abelian ideals. For instance, in every algebra from the family of $1357 \mathrm{M}$ there are (in addition to the above ideal $I_{4}^{\prime}=<e_{3}, e_{5}, e_{6}, e_{7}>$ ) also $I_{4}=<e_{4}, e_{5}, e_{6}, e_{7}>$ and $I_{4}^{\prime \prime}=<e_{2}, e_{3}, e_{5}, e_{7}>$.

\section{Degeneracy of the orbits of 7-dimensional Lie algebras}

A scheme for constructing realizations of 7-dimensional Lie algebras as the algebras of holomorphic vector fields in space $\mathbb{C}^{4}$ essentially repeats a similar scheme implemented in [1-4] for 5 -dimensional algebras. The main technical idea here is to simplify the form of basis vector fields of distinguished ideal (and then basis of the whole algebra under discussion). Algebras with simplified bases can be integrated (with overcoming certain technical difficulties).

So, write each element of the basis $e_{1}, \ldots, e_{7}$ of the discussed Lie algebra $\mathfrak{g}$ in the form of a holomorphic vector field in space $\mathbb{C}^{4}$ :

$$
e_{k}=a_{k}(z) \frac{\partial}{\partial z_{1}}+b_{k}(z) \frac{\partial}{\partial z_{2}}+c_{k}(z) \frac{\partial}{\partial z_{3}}+d_{k}(z) \frac{\partial}{\partial z_{4}} \quad(k=1, \ldots, 7) .
$$

In this entry, $a_{k}(z), b_{k}(z), c_{k}(z), d_{k}(z)$ are holomorphic (near the discussed point of the surface) functional coefficients, $z=\left(z_{1}, z_{2}, z_{3}, z_{4}\right)$ is a vector of complex coordinates. We will also use entries of the form $e_{k}=\left(a_{k}, b_{k}, c_{k}, d_{k}\right)$ to shorten the formula (10).

A real hypersurface $M=\{\Phi=0\}$ is the orbit (or integral surface) of a holomorphic realization of the algebra $\mathfrak{g}$ if for each base field $e_{k}$ of this algebra the condition of tangency $M$ is satisfied in the form

$$
\operatorname{Re}\left(\left.e_{k}(\Phi)\right|_{M}\right)=0
$$

Lemma 1. Let a real hypersurface $M \subset \mathbb{C}^{4}$ be Levy non-degenerate near some of its point $Q$ and let it be the orbit of the 7-dimensional Lie algebra $\mathfrak{g}$ of holomorphic vector fields in this space. Let also $I_{4}$ be a 4-dimensional Abelian subalgebra in $\mathfrak{g}$ with a fixed basis $e_{4}, e_{5}, e_{6}, e_{7}$.

This basis can be reduced to one of three forms by holomorphic change of coordinates of the space $\mathbb{C}^{4}$ (defined near the point $Q$ ):

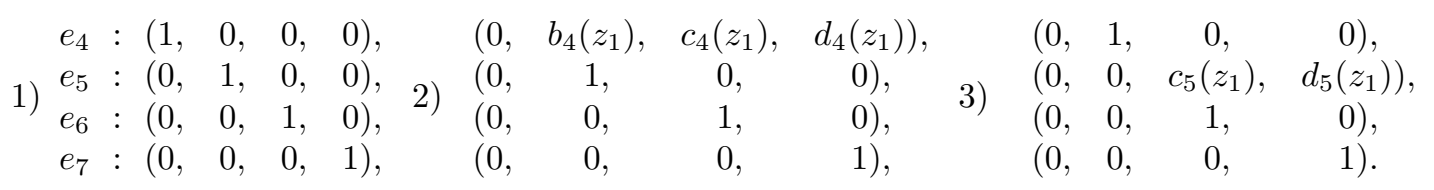


Remark 1. One can use the Abelian ideals mentioned above as Abelian subalgebras for the studied families of Lie algebras (4)-(9).

Proof of Lemma 1. First, we note that the assertion being proved is a simple generalization of Lemmas 3.1-3.4, formulated and proved in [4] for algebras of holomorphic vector fields in a 3 -dimensional complex space. As in [1,4], reduction the basis of the subalgebra to one of the desired types is possible by a step-by-step procedure.

For example, in the space $\mathbb{C}^{n}$ of any dimension $n$, a separate holomorphic vector field can be (locally) rectified, i.e. reduced by a holomorphic change of coordinates to differentiation with respect to one of the variables. In the situation discussed in Lemma 1, we bring thus the field $e_{7}$ to the form $\partial / \partial z_{4}=(0,0,0,1)$.

For another basis field $e_{6}$ of the subalgebra $I_{4}$ we consider its components $\left(a_{6}, b_{6}, c_{6}, d_{6}\right)$ in the new coordinates. Firstly, due to the commutation of the fields $e_{6}$ and $e_{7}$, these components are independent of the $z_{4}$ variable. And secondly, the truncated set $\left(a_{6}, b_{6}, c_{6}\right)$ cannot be identically zero, because a linear dependence over $\mathbb{C}$ (at each point of the surface $M$ ) of two fields $e_{6}$ and $e_{7}$ would mean Levy degeneracy of the discussed orbit.

Then the "truncated" vector field $\left(a_{6}\left(z_{1}, z_{2}, z_{3}\right), b_{6}\left(z_{1}, z_{2}, z_{3}\right), c_{6}\left(z_{1}, z_{2}, z_{3}\right)\right)$ can be (locally) straightened by a holomorphic change of three variables $z_{1}, z_{2}, z_{3}$. Accordingly, the field $e_{6}$ will take the form $\left(0,0,1, d_{6}\left(z_{1}, z_{2}, z_{3}\right)\right)$, and the field $e_{7}=(0,0,0,1)$ will remain rectified. The reduction of the field $e_{6}$ to the rectified form is completed (with preservation of the rectified field $e_{7}$ ) by another holomorphic change of coordinates $z_{4}^{*}=z_{4}+\varphi\left(z_{1}, z_{2}, z_{3}\right)$, with an arbitrary function $\varphi\left(z_{1}, z_{2}, z_{3}\right)$ satisfying the condition

$$
\partial \varphi / \partial z_{3}=-d_{6}\left(z_{1}, z_{2}, z_{3}\right) .
$$

Further, when passing to the simplification of the field $e_{5}$, several cases arise. A common fact for all such cases is independence of $e_{5}$ components of two variables $z_{3}, z_{4}$ (due to the commutation of $e_{5}$ with the fields $e_{6}, e_{7}$ ).

In the first case, with a nonzero "truncated" field $\left(a_{5}\left(z_{1}, z_{2}\right), b_{5}\left(z_{1}, z_{2}\right)\right)$ it can be straightened (just like the whole field $e_{5}$ ) due to operations similar to those described when rectifying the field $e_{6}$. The basis of the algebra $I_{4}$ takes then form

$\begin{array}{rllll}e_{4}: & \left(a_{4}\left(z_{1}\right),\right. & b_{4}\left(z_{1}\right), & c_{4}\left(z_{1}\right), & \left.d_{4}\left(z_{1}\right)\right), \\ e_{5} & :(0, & 1, & 0, & 0), \\ e_{6}: & :(0, & 0, & 1, & 0), \\ e_{7}: & :(0, & 0, & 0, & 1) .\end{array}$

Note that the components of the field $e_{4}$ in this situation can depend on no more than a single complex variable $z_{1}$ due to the commutation of all fields in $I_{4}$.

The second case is also possible, in which (after straightening the fields $e_{6}, e_{7}$ ) the basis of the subalgebra $I_{4}$ has the following coordinate representation

$$
\begin{array}{cccc}
e_{4}:\left(a_{4}\left(z_{1}, z_{2}\right),\right. & b_{4}\left(z_{1}, z_{2}\right), & c_{4}\left(z_{1}, z_{2}\right), & \left.d_{4}\left(z_{1}, z_{2}\right)\right), \\
e_{5}:(0, & 0, & c_{5}\left(z_{1}, z_{2}\right), & \left.d_{5}\left(z_{1}, z_{2}\right)\right), \\
e_{6}:(0, & 0, & 1, & 0), \\
e_{7}:(0, & 0, & 0, & 1) .
\end{array}
$$

Note also that in the case of identically zero components $a_{4}, b_{4}$, the four fields $e_{4}, \ldots, e_{7}$ turn out to be linearly dependent over $\mathbb{C}$ at each point on the surface $M$, and the equation of the 
surface (due to the tangency conditions for these fields) get rid of the variables $z_{1}, z_{2}$. Because in a Levy non-degenerate case this is impossible, the components $a_{4}, b_{4}$ cannot be both identically zero.

Now, using the inequality $\left(a_{4}\left(z_{1}, z_{2}\right), b_{4}\left(z_{1}, z_{2}\right)\right) \neq(0,0)$, we can, as in the discussion of the field $e_{6}$, straighten such a "truncated" field by a holomorphic change of two variables $z_{1}, z_{2}$. Then the field $e_{4}$ is rectified to the state $(0,1,0,0)$. The fields $e_{5}, e_{6}$ will retain under this their rectified appearance.

In this case, the whole base tetrad of the subalgebra $I_{4}$ acquires the third of the possible forms indicated in the lemma that we are proving. The first two possibilities arise when completing simplifications of a basis of the form (12).

If, for example, the coefficient $a_{4}\left(z_{1}\right)$ is not identically zero, then turning it into unity and after that straightening the field $e_{1}$ using the procedures already described, we get the "fully" rectified subalgebra $I_{4}$, i.e. case 1 ) from the lemma being proved. If $a_{4}\left(z_{1}\right) \equiv 0$, we get the form 2) for the basis of the algebra $I_{4}$. Lemma 1 is completely proved.

Further, it is proposed to consider for each algebra three possible cases from this lemma. Under this, one can significantly simplify such considerations using the following two statements.

Proposition 1. Suppose that a \%-dimensional real Lie algebra $\mathfrak{g}$ has a basis $e_{1}, \ldots, e_{7}$ with the following properties:

1) $I_{4}=<e_{4}, e_{5}, e_{6}, e_{7}>$ is an Abelian ideal in $\mathfrak{g}$;

2) for $h=<e_{1}, e_{2}, e_{3}>$ and $I_{4}$, the set of commutators $\left[h, I_{4}\right]$ is contained in the linear span $<e_{5}, e_{6}, e_{7}>$;

3) $\left[e_{1}, e_{2}\right]=e_{4}$.

Then a holomorphic realization of the algebra $\mathfrak{g}$ in the space $\mathbb{C}^{4}$ with the "straightened" ideal $I_{4}$ is impossible.

Proof. Suppose, on the contrary, that in the space $\mathbb{C}^{4}$ there exists a holomorphic realization of the algebra $\mathfrak{g}$ with a "straightened" basis of the ideal $I_{4}$, satisfying conditions 1$)-3$ ).

Consider a nonzero element $e_{1}$ of three-dimensional subspace $h$ of the Lie algebra $\mathfrak{g}$. Due to conditions 1)-2) of the proposition under discussion, we have

$$
\left[e_{1}, e_{4}\right]=0 \cdot e_{4}+A_{5} e_{5}+A_{6} e_{6}+A_{7} e_{7}=\left(0, A_{5}, A_{6}, A_{7}\right)
$$

with some real constants $A_{5}, A_{6}, A_{7}$.

But for a rectified holomorphic vector field $e_{4}$, the commutator $\left[e_{1}, e_{4}\right]$ is equal to $-\frac{\partial}{\partial z_{1}}\left(e_{1}\right)$.

This means that, by virtue of equality (13), the field $e_{1}$ can be represented in coordinates in the form

$$
e_{1}=\left(a_{1}\left(z_{2}, z_{3}, z_{4}\right),-A_{5} z_{1}+b_{1}\left(z_{2}, z_{3}, z_{4}\right),-A_{6} z_{1}+c_{1}\left(z_{2}, z_{3}, z_{4}\right),-A_{7} z_{1}+d_{1}\left(z_{2}, z_{3}, z_{4}\right)\right) .
$$

Similarly to (13), the commutators $\left[e_{1}, e_{5}\right],\left[e_{1}, e_{6}\right],\left[e_{1}, e_{7}\right]$, of the field $e_{1}$ with differentiations with respect to variables $z_{2}, z_{3}, z_{4}$ also do not contain $e_{4}$ in its expansions. This means that one can refine the form of the four functional coefficients

$$
\left(a_{1}\left(z_{2}, z_{3}, z_{4}\right), b_{1}\left(z_{2}, z_{3}, z_{4}\right), c_{1}\left(z_{2}, z_{3}, z_{4}\right), d_{1}\left(z_{2}, z_{3}, z_{4}\right)\right)
$$

in formula (14) and write the field $e_{1}$ as follows:

$$
e_{1}=\left(A_{1}, L_{12}\left(z_{1}, z_{2}, z_{3}, z_{4}\right)+B_{1}, L_{13}\left(z_{1}, z_{2}, z_{3}, z_{4}\right)+C_{1}, L_{14}\left(z_{1}, z_{2}, z_{3}, z_{4}\right)+D_{1}\right) .
$$


Here, all linear forms $L_{1 k}\left(z_{1}, z_{2}, z_{3}, z_{4}\right)(k=2,3,4)$ have only real coefficients, and $A_{1}, B_{1}, C_{1}, D_{1}$ are some complex constants.

It is clear that any vector field from the subspace $h$ has the form (15). Consider in such a situation a commutator of the field $e_{1}$ with a similar field

$$
e_{2}=\left(A_{2}, L_{22}\left(z_{1}, z_{2}, z_{3}, z_{4}\right)+B_{2}, L_{23}\left(z_{1}, z_{2}, z_{3}, z_{4}\right)+C_{2}, L_{24}\left(z_{1}, z_{2}, z_{3}, z_{4}\right)+D_{2}\right)
$$

It is clear that the $z_{1}$-component of such a commutator is zero, which contradicts condition 3 ) of the proposition under discussion. Consequently, this proposition is proved.

Proposition 2. For each of the discussed algebras of all six families (4)-(9) there are bases satisfying conditions 1)-3) of Proposition 1.

Proof. The $1357 M$ and $1357 Q R S_{1}$ families contain the ideal $I_{4}=<e_{3}, e_{5}, e_{6}, e_{7}>$. Under this decomposition $\left[e_{1}, I_{4}\right],\left[e_{2}, I_{4}\right],\left[e_{4}, I_{4}\right]$ do not contain $e_{3}$ component, and $\left[e_{1}, e_{2}\right]=e_{3}$.

In the $1357 N$ family with the same ideal $I_{4}=<e_{3}, e_{5}, e_{6}, e_{7}>$ the expansions $\left[e_{1}, I_{4}\right],\left[e_{2}, I_{4}\right]$, $\left[e_{4}, I_{4}\right]$ do not contain $e_{6}$ components, and $\left[e_{1}, e_{4}\right]=e_{6}$.

Finally, the families $12457 \mathrm{~N}, 123457 \mathrm{I}, 12457 \mathrm{~N}_{2}$ have the same structure from the point of view interesting to us: the 4-dimensional Abelian ideal in algebras from these families is $I_{4}=<e_{4}, e_{5}, e_{6}, e_{7}>$; decompositions of $\left[e_{1}, I_{4}\right],\left[e_{2}, I_{4}\right],\left[e_{3}, I_{4}\right]$ do not contain $e_{4}$-component and at the same time $\left[e_{1}, e_{3}\right]=e_{4}$. Proposition 2 is proved.

Recall that the main interest of the paper is related to Levi non-degenerate orbits of nilpotent Lie algebras. Application of Propositions 1 and 2 to each of the six families of algebras (4)-(9) (with the basis vectors ordering in 4-dimensional ideals corresponding to the Proposition 2) allows in all cases to reduce the meaningful discussions to the points 2) and 3) of Lemma 1.

\section{An analogy with the case of 5-dimensional algebras}

Proposition 3. Realizations of the algebras $1357 \mathrm{~N}, 12457 \mathrm{~N}, 123457 \mathrm{I}, 12457 \mathrm{~N}_{2}$ with the fixed bases of their Abelian ideals simplified to types 2) or 3) can have only Levi-degenerate orbits in the space $\mathbb{C}^{4}$.

Remark. We discuss Proposition 3 only for the $12457 N$-family described by the maximal number of nontrivial commutation relations. The three remaining families (with the corresponding renumbering of the base fields) can be considered similarly.

Proof. Proposition 1 prohibits the existence of Levi-non-degenerate orbits for this family in case 1 ) of Lemma 1. We show that the 21 commutation relations (10 of which are trivial) in the 7 -dimensional Lie algebra of the $12457 N$-family also contradict cases 2) and 3) of this lemma.

In case 2) of Lemma 1 we have a triple of rectified fields

$$
e_{5}=(0,1,0,0), e_{6}=(0,0,1,0), e_{7}=(0,0,0,1)
$$

and the field $e_{4}=\left(0, b_{4}\left(z_{1}\right), c_{4}\left(z_{1}\right), d_{4}\left(z_{1}\right)\right)$.

We note, first, that the six pairwise relations for the four basis fields of the ideal have already been used (verified) for the obtaining a simplified form of the basis of the ideal $I_{4}$. Second, consideration of nine commutators of each of the triple rectified fields $e_{5}, e_{6}, e_{7}$ with each of the three fields $e_{1}, e_{2}, e_{3}$ from the complement to the ideal $I_{4}$ allows as to we get a simplified form 
of these additional fields:

$$
\begin{aligned}
& e_{1}=\left(a_{1}\left(z_{1}\right), b_{1}\left(z_{1}\right),-z_{2}+c_{1}\left(z_{1}\right),-z_{3}+d_{1}\left(z_{1}\right)\right), \\
& e_{2}=\left(a_{2}\left(z_{1}\right), b_{2}\left(z_{1}\right), c_{2}\left(z_{1}\right),-z_{3}-\lambda z_{2}+d_{2}\left(z_{1}\right)\right), \\
& e_{3}=\left(a_{3}\left(z_{1}\right), b_{3}\left(z_{1}\right), c_{3}\left(z_{1}\right), z_{2}+d_{3}\left(z_{1}\right)\right) .
\end{aligned}
$$

Next, we move on to more "subtle" verification actions. For example, by coordinate-wise writing the relation $\left[e_{2}, e_{4}\right]=e_{6}$, we get

$$
a_{2}\left(0, b_{4}^{\prime}\left(z_{1}\right), c_{4}^{\prime}\left(z_{1}\right), d_{4}^{\prime}\left(z_{1}\right)\right)-\left(b_{4}(0,0,0,-\lambda)+c_{4}(0,0,0,-1)\right)=(0,0,1,0) .
$$

The second and the third components of this vector equality have the form

$$
a_{2}\left(z_{1}\right) \cdot b_{4}^{\prime}\left(z_{1}\right)=0, \quad a_{2}\left(z_{1}\right) \cdot c_{4}^{\prime}\left(z_{1}\right)=1 .
$$

This means that $a_{2}\left(z_{1}\right)$ is nonzero (near the origin). In this situation, one can use the "linearization lemma" proved in [1] (see also [4], Remark 3.2). This lemma, applied to the field $e_{2}$, allows us to bring it after a holomorphic change of coordinates to the form

$$
e_{2}=\left(1,0,0,-z_{3}-\lambda z_{2}\right)
$$

instead of the more complicated form fixed in formulas (17). The rectified fields $e_{5}, e_{6}, e_{7}$ will be preserved; the fields $e_{1}, e_{3}, e_{4}$ will also retain their simplified structure.

Considering further the equalities $\left[e_{1}, e_{4}\right]=\left[e_{3}, e_{4}\right]=e_{7}$, one can obtain the next simplifications of the first four basis fields:

$$
\begin{aligned}
& e_{1}=\left(1, b_{1}\left(z_{1}\right),-z_{2}+c_{1}\left(z_{1}\right),-z_{3}+d_{1}\left(z_{1}\right)\right), \quad e_{2}=\left(1,0,0,-z_{3}-\lambda z_{2}\right), \\
& e_{3}=\left(0, b_{3}\left(z_{1}\right), c_{3}\left(z_{1}\right), z_{2}+d_{3}\left(z_{1}\right)\right), \quad e_{4}=\left(0,-1, \frac{1-\lambda}{2}, \frac{1+\lambda}{2} z_{1}+D_{4}\right),
\end{aligned}
$$

where $D_{4}$ is a complex constant.

Finally, we check the remaining three commutation relations

$$
\left[e_{1}, e_{2}\right]=e_{3}, \quad\left[e_{1}, e_{3}\right]=e_{4}, \quad\left[e_{2}, e_{3}\right]=e_{5} .
$$

The last of them contains restrictions on the field $e_{3}$, which now takes the form

$$
e_{3}=\left(0, z_{1}+B_{3}, C_{3}, z_{2}-\frac{\lambda}{2}\left(z_{1}+B_{3}\right)^{2}+C_{3}\left(z_{1}+B_{3}\right)+D_{3}\right)
$$

with arbitrary complex constants $B_{3}, C_{3}, D_{3}$.

Then the first of relations (19) leads to a rather complicated form of the field

$$
e_{1}=\left(1,-\frac{1}{2}\left(z_{1}+B_{3}\right)^{2}+B_{1},-z_{2}+\left(-C_{3} z_{1}+C_{1}\right),-z_{3}-\frac{\lambda}{3}\left(z_{1}+B_{3}\right)^{3}-N z_{1}+D_{1}\right),
$$

where $N=\left(\lambda B_{1}+C_{2} B_{3}+D_{3}-C_{1}\right)$, and $B_{1}, C_{1}, D_{1} \in \mathbb{C}$ are arbitrary constants.

Taking into account formulas (20), (21), the left-hand side of the second relations (19) can be written in a form

$$
\begin{aligned}
{\left[e_{1}, e_{3}\right]=} & \left(\left(0,1,0,-\lambda\left(z_{1}+B_{3}\right)+C_{3}\right)+\left(-\frac{1}{2}\left(z_{1}+B_{3}\right)^{2}+B_{1}\right) \cdot(0,0,0,1)\right)- \\
& -\left(\left(z_{1}+B_{3}\right) \cdot(0,0,-1,0)+C_{3}(0,0,0,-1)\right) .
\end{aligned}
$$


The second component of this vector field equals to 1 , contrary to the fact that the equal field $e_{4}$ has -1 in the second component. Thus, in the second case of Lemma 1, algebras of the family $123457 \mathrm{~N}$ do not admit holomorphic realizations.

Now discussing case 3) of Lemma 1, we have:

$$
\begin{aligned}
& e_{4}:(0,1, \quad 0, \quad 0) \\
& e_{5}:\left(0,0, \quad c_{5}\left(z_{1}\right), d_{5}\left(z_{1}\right)\right)
\end{aligned}
$$

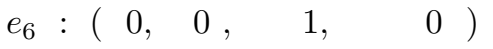

$$
\begin{aligned}
& e_{7}:\left(\begin{array}{llll}
0, & 0, & 0, & 1
\end{array}\right)
\end{aligned}
$$

Using the nine commutation relations $\left[e_{1}, e_{4}\right]=e_{7},\left[e_{1}, e_{6}\right]=e_{7},\left[e_{1}, e_{7}\right]=0$,

$$
\left[e_{2}, e_{4}\right]=e_{6},\left[e_{2}, e_{6}\right]=e_{7},\left[e_{2}, e_{7}\right]=0, \quad\left[e_{3}, e_{4}\right]=e_{7},\left[e_{3}, e_{6}\right]=0,\left[e_{3}, e_{7}\right]=0,
$$

we obtain formulas

$$
\begin{aligned}
& e_{1}=\left(a_{1}\left(z_{1}\right), b_{1}\left(z_{1}\right), c_{1}\left(z_{1}\right),-z_{2}-z_{3}+d_{1}\left(z_{1}\right)\right), \\
& e_{2}=\left(a_{2}\left(z_{1}\right), b_{2}\left(z_{1}\right),-z_{2}+c_{2}\left(z_{1}\right),-z_{3}+d_{2}\left(z_{1}\right)\right), \\
& e_{3}=\left(a_{3}\left(z_{1}\right), b_{3}\left(z_{1}\right), c_{3}\left(z_{1}\right),-z_{2}+d_{3}\left(z_{1}\right)\right),
\end{aligned}
$$

similar to the collection (18) from the case 2 .

We now note that in the case of identical vanishing of any two of the three coefficients $a_{1}\left(z_{1}\right), a_{2}\left(z_{1}\right), a_{3}\left(z_{1}\right)$ the six basic fields of the algebra under discussion turn out to be linearly dependent over $\mathbb{C}$. This leads to Levi degeneration of all orbits of the Lie algebra with a basis of the form $(22)-(23)$.

Therefore, the search for algebras with such bases admitting non-degenerate orbits can be reduced to two subcases:

subcase 3.1. $a_{3}\left(z_{1}\right) \neq 0$;

subcase 3.2. $a_{3}\left(z_{1}\right) \equiv 0, a_{1}\left(z_{1}\right) \neq 0$.

Applying the linearization lemma in each of these subcases, we can, in addition to the rectified triple of fields from (22), significantly simplify one another field. In subcase 3.1, we can regard the field $e_{3}$ having a simplified form $e_{3}=\left(1,0,0,-z_{2}\right)$, and in subcase 3.2 we have

$$
e_{1}=\left(1,0,0,-z_{2}-z_{3}\right), \quad e_{3}=\left(0, b_{3}\left(z_{1}\right), c_{3}\left(z_{1}\right),-z_{2}+d_{3}\left(z_{1}\right)\right)
$$

Given this simplification, we consider in subcase 3.1 three commutation relations $\left[e_{1}, e_{3}\right]=0$, $\left[e_{2}, e_{3}\right]=e_{5},\left[e_{1}, e_{2}\right]=e_{3}$.

The first of these relations has the expanded form

$$
b_{1}(0,0,0,-1)-\left(a_{1}^{\prime}, b_{1}^{\prime}, c_{1}^{\prime}, d_{1}^{\prime}\right)=(0,0,0,0) .
$$

It means that $e_{1}=\left(A_{1}, B_{1}, C_{1},-z_{2}-z_{3}-B_{1} z_{1}+D_{1}\right)$ with some complex constants $A_{1}, B_{1}, C_{1}, D_{1}$. Similarly, from $\left[e_{2}, e_{3}\right]=e_{5}$ we get

$$
e_{2}=\left(A_{2}, B_{2},-z_{2}+c_{2}\left(z_{1}\right),-z_{3}+d_{2}\left(z_{1}\right)\right), \quad e_{5}=\left(0,0,-c_{2}^{\prime}\left(z_{1}\right),-d_{2}^{\prime}\left(z_{1}\right)-B_{2}\right)
$$

with complex constants $A_{2}, B_{2}$ and holomorphic functions $c_{2}\left(z_{1}\right), d_{2}\left(z_{1}\right)$.

But taking into account the formulas obtained for the fields $e_{1}, e_{2}$, the first component of the commutator

$$
\begin{aligned}
{\left[e_{1}, e_{2}\right] } & =\left(A_{1}\left(0,0, c_{2}^{\prime}\left(z_{1}\right), d_{2}^{\prime}\left(z_{1}\right)\right)+B_{1}(0,0,-1,0)+C_{1}(0,0,0,-1)\right)- \\
& -\left(A_{2}\left(0,0,0,-B_{1}\right)+B_{2}(0,0,0-1)+\left(-z_{2}+c_{2}\left(z_{1}\right)\right)(0,0,0,-1)\right)
\end{aligned}
$$


is zero, contrary to the fact that $e_{3}=\left(1,0,0,-z_{2}\right)$.

A similar contradiction also arises in subcase 3.2. Here, from the equality $\left[e_{1}, e_{3}\right]=0$ we obtain the formula

$$
e_{3}=\left(0, B_{3}, C_{3},-z_{2}-\left(B_{3}+C_{3}\right) z_{1}+D_{3}\right) .
$$

Then a refined form of the field $e_{2}$ is derived from the relation $\left[e_{1}, e_{2}\right]=e_{3}$ :

$$
e_{2}=\left(A_{2}, B_{3} z_{1}+B_{2},-z_{2}+C_{3} z_{1}+C_{2},-z_{3}+\left(B_{3}+C_{3}\right) z_{1}^{2}+\left(D_{3}-B_{2}-C_{2}\right) z_{1}+D_{2}\right) .
$$

And the field $e_{5}$, equal to the commutator of these two fields, will now take the form

$$
e_{5}=\left(0,0, B_{3},-B_{3} z_{1}+\left(-C_{3}-B_{2}-A_{2} B_{3}-A_{2} C_{3}\right)\right) .
$$

In this subcase, the last three commutation relations were not considered: $\left[e_{1}, e_{5}\right]=e_{6},\left[e_{2}, e_{5}\right]=$ $=\lambda e_{7},\left[e_{3}, e_{5}\right]=-e_{7}$.

Taking into account formulas (24) and (25), the first of them leads to a contradiction, since

$$
\left[e_{1}, e_{5}\right]=\left(0,0,0,-B_{3}\right)-B_{3}(0,0,0,-1)=(0,0,0,0) \neq e_{6} .
$$

Proposition 3 for the family of Lie algebras $12457 N$ is proved.

\section{Integration of the $1357 M$ and $1357 Q R S 1$ families}

The technique of the previous section allows us to obtain similar conclusions in the study of other algebras. The description of all possible holomorphic realizations of algebras from the families $1357 M$ and $1357 Q R S 1$ was received exactly in such manner. We omit here technical details of reasonings (close to fragments of Section 3) and note only the following two points.

1) The descriptions of holomorphic realizations of the two families $1357 M$ and $1357 Q R S 1$ almost coincide, because descriptions (4) and (8) of these families themselves have the only difference: the trivial commutator $\left[e_{2}, e_{3}\right]=0$ in the family $1357 M$ is replaced by a nontrivial relation $\left[e_{2}, e_{3}\right]=-e_{6}$ for the $1357 Q R S 1$ family.

2) This description is connected with the ideal $I_{4}^{\prime}=\left\langle e_{4}, e_{5}, e_{6}, e_{7}\right\rangle$, which is most often found in the six (and even in nine) algebra families under consideration, but not with the ideal $I_{4}=<e_{3}, e_{5}, e_{6}, e_{7}>$ distinguished in Proposition 2. Thereby, all three cases of Lemma 1 were directly verified, while Proposition 1 was not used.

\begin{tabular}{|c|c|c|c|}
\hline $\begin{array}{l}e_{1}:(1, \\
e_{2}:\left(-\lambda C_{5},\right.\end{array}$ & $\begin{array}{c}0, \\
B_{3} z_{1}+B_{2},\end{array}$ & $\begin{array}{c}-z_{2} \\
c_{2}\left(z_{1}, z_{2}\right)\end{array}$ & $\begin{array}{c}0 \\
d_{2}\left(z_{1}, z_{2}, z_{3}\right)\end{array}$ \\
\hline$e_{3}:($ & $B_{3}$ & $\left(C_{5}-B_{3}\right) z_{1}+C_{3}$ & $\left.z_{1}^{2} / 2+D_{5} z_{1}+(\lambda-1) z_{2}+D_{3}\right)$ \\
\hline$e_{4}:($ & 1 & 0 & 0 \\
\hline$e_{5}:($ & 0 & $C_{5}$ & $z_{1}+D_{5}$ \\
\hline$e_{6}:($ & 0 & 1, & 0 \\
\hline$e_{7}:($ & 0 & 0 & 1 \\
\hline
\end{tabular}

Proposition 4. Holomorphic realizations of the families $1357 M, 1357 Q R S 1$ connected with the ideal $I_{4}^{\prime}=<e_{4}, e_{5}, e_{6}, e_{7}>$ and admitting Levi non-degenerate orbits are possible only in case 3) of Lemma 1. The bases of such realizations have the form $\left(\operatorname{Im} B_{3} \neq 0, \operatorname{Im} C_{5} \neq 0\right)$ : 
where

$$
\begin{gathered}
c_{2}\left(z_{1}, z_{2}\right)=\left(C_{5}-2 B_{3}\right) z_{1}^{2} / 2+\left(C_{3}-B_{2}\right) z_{1}-C_{5} z_{2}+C_{2}, \\
d_{2}\left(z_{1}, z_{2}, z_{3}\right)=z_{1}^{3} / 6+D_{5} z_{1}^{2} / 2+D_{3} z_{1}-\left(z_{1}+D_{5}\right) z_{2}-\lambda z_{3}+D_{2} .
\end{gathered}
$$

Conditions for the $1357 M$ family:

$$
-\lambda C_{5}+(\lambda+1) B_{3}=0, \quad-\lambda C_{5} D_{5}+B_{2}(\lambda-1)+B_{3} D_{5}+C_{3} \lambda=0 .
$$

Conditions for the 1357QRS1 family:

$$
-\lambda C_{5}\left(C_{5}-B_{3}\right)+B_{3} C_{5}=-1, \quad-\lambda C_{5} D_{5}+B_{2}(\lambda-1)+B_{3} D_{5}+C_{3} \lambda=0 .
$$

To complete the proof of Theorem 2, it remains to integrate the algebras of vector fields, obtained in Proposition 5 and present non-degenerate nonspherical orbits of these algebras.

Recall that the defining function for the orbit of an arbitrary algebra of holomorphic vector fields with basis $e_{1}, \ldots, e_{7}$ in $\mathbb{C}^{4}$ is a solution to a system of seven partial differential equations of the form (11) for $k=1, \ldots, 7$.

Given the presence in the realizations of all the algebras discussed, the triple of rectified fields and being interested only in non-degenerate orbits, one can consider that each of them is described by an equation of the form

$$
y_{4}=F\left(x_{1}, y_{1}, y_{2}, y_{3}\right)
$$

But even with such simplification the integration of the system of (only) four equations presents, generally speaking, considerable technical difficulties.

Proposition 5. For $\lambda \neq-1$ Levi non-degenerate orbits of algebras with bases (26) from the family of $1357 M$ are (up to local holomorphic transformations) only algebraic tubular surfaces with affine-homogeneous bases

$$
y_{4}=y_{1} y_{3}+A y_{2}^{2}+B y_{1}^{2} y_{2}+C y_{1}^{4},
$$

where

$$
A=\frac{1-\lambda}{2(1+\lambda)}, \quad B=\frac{1}{1+\lambda}, \quad C=\frac{1}{4(1+\lambda)} .
$$

Proof. We use another Abelian ideal $I_{4}^{\prime \prime}=<e_{2}, e_{3}, e_{5}, e_{7}>$ in the algebras of the $1357 M$ family. Coordinate description in $\mathbb{C}^{4}$ of the basis of this ideal (in the holomorphic realization from Proposition 4) is upper triangular:

$$
\left.\begin{array}{rlccc}
e_{2} & :\left(-\lambda C_{5},\right. & B_{3} z_{1}+B_{2}, & c_{2}\left(z_{1}, z_{2}\right) & d_{2}\left(z_{1}, z_{2}, z_{3}\right) \\
e_{3}:( & 0, & B_{3}, & \left(C_{5}-B_{3}\right) z_{1}+C_{3}, & \left.z_{1}^{2} / 2+D_{5} z_{1}+(\lambda-1) z_{2}+D_{3}\right), \\
e_{5}:( & 0, & 0, & C_{5}, & z_{1}+D_{5} \\
e_{7}:( & 0, & 0, & 0, & 1
\end{array}\right),
$$

Moreover, $\operatorname{Im} B_{3} \neq 0, \operatorname{Im} C_{3} \neq 0$. Then after complex dilation of variables

$$
z_{1}=-\lambda C_{5} z_{1}^{*}, \quad z_{2}=B_{3} z_{2}^{*}, \quad z_{3}=C_{5} z_{3}^{*}
$$

each of the four diagonal elements of the matrix (29) will be equal to unity. 
We apply to the fields (29) the sequential straightening procedure described in Section 2 above using the commutation of any pair of these fields. Then the basis of the $1357 M$ family will take much simpler affine form

$$
\begin{aligned}
& e_{1}=i \frac{\partial}{\partial z_{1}}-z_{1} \frac{\partial}{\partial z_{2}}-z_{2} \frac{\partial}{\partial z_{3}}-z_{3} \frac{\partial}{\partial z_{4}}, \quad e_{2}=\frac{\partial}{\partial z_{1}}, \quad e_{3}=\frac{\partial}{\partial z_{2}} \\
& e_{4}=-i(1+\lambda) \frac{\partial}{\partial z_{2}}+z_{1} \frac{\partial}{\partial z_{3}}+(1-\lambda) z_{2} \frac{\partial}{\partial z_{4}} \\
& e_{5}=\frac{\partial}{\partial z_{3}}, \quad e_{6}=-i \lambda \frac{\partial}{\partial z_{3}}+\lambda z_{1} \frac{\partial}{\partial z_{4}}, \quad e_{7}=\frac{\partial}{\partial z_{4}} .
\end{aligned}
$$

For $\lambda \neq-1$, integration of an algebra with such a simplified basis leads precisely to equation (27) with coefficients of the form (28).

Note also that by dilation the variables

$$
z_{1}^{*}=t z_{1}, \quad z_{2}^{*}=r t_{2}, \quad z_{3}^{*}=s z_{3}, \quad z_{4}^{*}=q z_{4}
$$

with real coefficients $t, r, s, q$ the three nonzero coefficients $(A, B, C)$ from equation (27) can be transformed to the form $\left(1,1, A C / B^{2}\right)$.

With this in mind, for $\lambda \neq 1$, equation (27)-(28) can be reduced to

$$
y_{4}=y_{1} y_{3}+y_{2}^{2}+y_{1}^{2} y_{2}+D y_{1}^{4}
$$

where $D=C A / B^{2}=(1-\lambda) / 8$.

Concluding the proof of Proposition 5, we note that the quadratic form $y_{1} y_{3}+y_{2}^{2}$ from the right-hand side of equation (31) turns in complex coordinates into indefinite nondegenerate Levi form

$$
H\left(z_{1}, z_{2}, z_{3}\right)=z_{1} \bar{z}_{3}+z_{3} \bar{z}_{1}+\left|z_{2}\right|^{2} .
$$

Therefore, for $\lambda \neq \pm 1$ all the orbits of (4) are non-degenerate. Proposition 5 is proved.

Remark 1. For $\lambda=-1$, all orbits of such an algebra in the space $\mathbf{C}^{4}$ are affine equivalent to a Levy degenerate hypersurface $y_{1}=y_{2}^{2}$.

Remark 2. According to [8], the surface with equation (4) is spherical, i.e. holomorphically equivalent to the corresponding quadric

$$
y_{4}=z_{1} \bar{z}_{3}+z_{3} \bar{z}_{1}+\left|z_{2}\right|^{2},
$$

only for $A=1 / 12$, i.e. with $\lambda=1 / 3$.

Thus, the first part of Theorem 2 is proved, and the family of surfaces (4) illustrates the difference between the situation in $\mathbf{C}^{4}$ and the 3 -dimensional case.

Remark 3. For $\lambda=1$, the surface (27)-(28), i.e.

$$
y_{4}=y_{1}\left(y_{3}+\frac{1}{2} y_{1} y_{2}+\frac{1}{8} y_{1}^{3}\right) .
$$

is Levi degenerate.

Remark 4. Any surface from family (31) admits a consistent expansion of variables that preserves both the surface and the origin of $\mathbf{C}^{4}$ lying on them. This means that an algebra with basis (30) is a subalgebra of an 8-dimensional algebra, the additional basis field of which is

$$
e_{8}=z_{1} \frac{\partial}{\partial z_{1}}+2 z_{2} \frac{\partial}{\partial z_{2}}+3 z_{3} \frac{\partial}{\partial z_{3}}+4 z_{4} \frac{\partial}{\partial z_{4}} .
$$

The following statement completes the proof of Theorem 2 . 
Proposition 6. For arbitrary $\lambda>1$ and for the following parameter values

$$
B_{3}=C_{5}=i, D_{5}=\frac{\lambda}{\lambda-1}, B_{2}=C_{3}=D_{3}=0
$$

the orbits of any algebra from the family $1357 Q R S_{1}$ with a basis of the form (26) are affinely equivalent to the surfaces

$$
y_{4}=y_{1} y_{3}+y_{2}^{2}+x_{1} y_{1} y_{2}+\frac{5}{48(\lambda-1)} y_{1}^{4}
$$

Proof of Proposition 6. Let us consider a system of partial differential equations corresponding to an algebra from family $1357 Q R S_{1}$. Due to the specified restrictions on the parameters four meaningful equations for the determining function of the surface $y_{4}=F\left(x_{1}, y_{1}, y_{2}, y_{3}\right)$ acquires a relatively simple form:

$$
\begin{aligned}
\frac{\partial F}{\partial x_{1}}-y_{2} \frac{\partial F}{\partial y_{3}}=0, \quad \frac{\partial F}{\partial y_{2}}=x_{1} y_{1}+\frac{\lambda}{\lambda-1} y_{1}+(\lambda-1) y_{2}, \quad \frac{\partial F}{\partial y_{3}}=y_{1}, \\
-\lambda \frac{\partial F}{\partial y_{1}}+x_{1} \frac{\partial F}{\partial y_{2}}+\left(-\frac{1}{2}\left(x_{1}^{2}-y_{1}^{2}\right)-x_{2}\right) \frac{\partial F}{\partial y_{3}}= \\
=\left(\frac{1}{2} x_{1}^{2} y_{1}+\frac{\lambda}{\lambda-1} x_{1} y_{1}-\frac{1}{6} y_{1}^{3}\right)-\left(\left(x_{1}+\frac{\lambda}{\lambda-1}\right) y_{2}+y_{1} x_{2}\right)-\lambda y_{3} .
\end{aligned}
$$

A step-by-step solution of the individual equations of this system leads to its final solution of the form

$$
F=y_{1} y_{3}+\frac{1}{2}(\lambda-1) y_{2}^{2}+y_{1} y_{2}\left(x_{1}+\mu\right)-\frac{5}{24} y_{1}^{4}+C, \quad C=\text { const }, \quad \mu=\frac{\lambda}{\lambda-1} .
$$

Due to affine transformations and, in particular, consistent coordinate expansion of the complex space $\mathbb{C}^{4}$, the equations of the desired orbits in this case can be written in the form (3), which is very close in form to (2). Proposition 6 and Theorem 2 are proved.

Remark 1. It is also possible to write out and integrate the equations corresponding to algebras from the family $1357 Q R S_{1}$ with arbitrary parameter values (the authors did this using the Maple package). The resulting equations of the orbits are very cumbersome, but in all non-degenerate cases they are reduced by holomorphic transformations to equations (3).

Remark 2. Currently, the authors are not aware of the answer to the question of holomorphic equivalence (or nonequivalence) for the surfaces (2) and (3). In multidimensional complex analysis, the task of practical verification of holomorphic equivalence of specific varieties is often hard enough to solve. Therefore, the study of the (possibly simple) question about the surfaces (2) and (3) can be be considered as going beyond the scope of this article.

This work was supported by the Russian Foundation for Basic Research (projects no. 17-0100592, 20-01-00497).

\section{References}

[1] V.K.Beloshapka, I.G.Kossovskiy, J. Geom. Anal., 20(2010), no. 3, 538-564.

DOI: $10.1007 / \mathrm{s} 12220-010-9117-4$

[2] R.S.Akopyan, A.V.Loboda, Funct. Anal. Its Appl., 53(2019), 124-128.

DOI: $10.1134 /$ S0016266319020059 
[3] A.V.Atanov, I.G.Kossovskyi, A.V.Loboda, Dokl. Math., 100(2019), 377-379. DOI: $10.1134 /$ S1064562419040173

[4] A.V.Atanov, A.V.Loboda, Decomposable five-dimensional Lie algebras in the holomorphic homogeneity problem in $\mathbb{C}^{3}$, Itogi nauki i tehniki. Sovrem. matem. i ee prilozh., 173(2019), 86-115 (in Russian).

[5] G.M.Mubarakzjanov, Classification of real structures of Lie algebras of fifth order, Izv. Vyssh. Uchebn. Zaved. Matematika, 34(1963), no. 3, 99-106 (in Russian).

[6] Ming-Peng Gong, Classification of Nilpotent Lie Algebras of Dimension 7, University Waterloo, Canada, 1998.

[7] V.V.Morozov, Classification of nilpotent Lie algebras of 6-th order, Izv. Vyssh. Uchebn. Zaved. Matematika, (1958), no. 4, 161-171 (in Russian).

[8] A.V.Isaev, M.A.Mishchenko, Mathematics of the USSR-Izvestiya, 33(1989), no. 3, 441-472. DOI: 10.1070/IM1989v033n03ABEH000852

\title{
Об орбитах нильпотентных 7-мерных алгебр Ли в 4-мерном комплексном пространстве
}

\author{
Александр В. Лобода \\ Воронежский государственный технический университет \\ Воронеж, Российская Федерация \\ Рипсиме С. Акопян \\ МИРЭА, Российский технологический университет \\ Москва, Российская Федерация \\ Владислав В. Крутских \\ Воронежский государственный университет \\ Воронеж, Российская Федерация
}

\begin{abstract}
Аннотация. В работе изучены однопараметрические семейства 7-мерных нильпотентных неразложимых алгебр Ли и орбиты голоморфных реализаций таких алгебр в 4-мерном комплексном пространстве. Показано, что в отличие от орбит 5-мерных нильпотентных алгебр Ли в 3-мерном пространстве два таких семейства (из имеющихся девяти) допускают орбиты, являющиеся невырожденными по Леви (однородными) вещественными несферическими гиперповерхностями. С точностью до голоморфной эквивалентности все полученные невырожденные несферические орбиты являются графиками многочленов 4-й степени.
\end{abstract}

Ключевые слова: алгебра Ли, комплексное пространство, векторное поле, голоморфная функция, однородное многообразие, вырождение по Леви. 